\title{
Use of a new availability index to evaluate the effect of policy changes to the Special Supplemental Nutrition Program for Women, Infants, and Children (WIC) on the food environment in New Orleans
}

\author{
Keelia O'Malley*, Brian G Luckett, Lauren Futrell Dunaway, J Nicholas Bodor and \\ Donald Rose \\ Department of Global Community Health and Behavioral Sciences, Tulane University School of Public Health and \\ Tropical Medicine (SPHTM), 1440 Canal Street, Suite 2301, New Orleans, LA 701 12, USA
}

Submitted 21 August 2013: Final revision received 30 January 2014: Accepted 10 March 2014: First published online 24 April 2014

\begin{abstract}
Objective: Changes to the Special Supplemental Nutrition Program for Women, Infants, and Children (WIC) occurred in 2009 when supplemental foods offered through the programme were updated to align with current dietary recommendations. The present study reports on a new index developed to monitor the retail environment's adoption of these new food supply requirements in New Orleans. Design: A 100-point WIC Availability Index (WIC-AI) was derived from new minimum state stocking requirements for WIC vendors. A sample of supermarkets, medium and small food stores was assessed in 2009 before changes were implemented and in 2010 after revisions had gone into effect. WIC-AI scores were utilized to compare differences in meeting requirements by store type, WIC vendor status and year of measurement.

Setting: Supermarkets, medium and small WIC and non-WIC food stores in New Orleans, Louisiana, USA.

Results: At baseline supermarkets had the highest median WIC-AI score (93.3) followed by medium (69.8) and small food stores (48.0). Small WIC stores had a higher median WIC-AI score at baseline than small non-WIC stores (66.9 v. 38.0). Both medium and small WIC stores significantly increased their median WIC-AI scores between 2009 and $2010(P<0 \cdot 01)$. The increased median WIC-AI score in small food stores was largely attributed to increased availability of cereals and grains, juices and fruit, and infant fruit and vegetables.

Conclusions: The WIC-AI is a simple tool useful in summarizing complex food store environment data and may be adapted for use in other states or a national level to inform food policy decisions and direction.
\end{abstract}

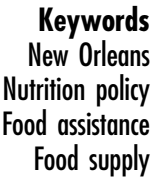

Keywords Nutrition policy Food supply
The Special Supplemental Nutrition Program for Women, Infants, and Children (WIC) was originally established in the 1970s as a response to the issue of malnutrition in the USA, particularly among vulnerable populations such as pregnant women and children ${ }^{(1)}$. Grants to operate the programme are provided to state agencies from the federal US Department of Agriculture's Food and Nutrition Service. State WIC agencies authorize vendors who are geographically distributed across their service area and also establish minimum stocking requirements for their authorized vendors ${ }^{(1)}$. Potential WIC recipients must be pregnant, breast-feeding or less than 5 years of age, meet income requirements and be considered at nutritional risk by a health professional to qualify to receive WIC benefits $^{(1)}$. In addition to nutrition education and referrals to health care, WIC participants receive monthly vouchers that are redeemed for food items included as part of a WIC food package at one of the authorized WIC food store vendors $^{(2)}$. WIC has demonstrated positive effects on the birth outcomes of prenatal WIC participants and positive effects on the food and nutrient intakes of participant children $^{(3-5)}$. The programme has expanded over the years and currently serves over 10 million women and children ${ }^{(6)}$. While malnutrition and food security remain an issue with $14.5 \%$ of American households experiencing food insecurity in $2010^{(7)}$, the most recent estimates indicate that $68.0 \%$ of American adults $^{(8)}$ and $31.8 \%$ of American children ${ }^{(9)}$ are overweight or obese. A combination of factors including advances in nutrition information, changes in dietary patterns, expansion of the WIC programme and increased 
public health concern related to overweight and obesity led to the 2006 Institute of Medicine report, WIC Food Packages: Time for a Change. Within the report, the Institute of Medicine laid out recommendations to revise the WIC food packages to align their contents more closely with current dietary guidelines ${ }^{(2)}$.

The first substantial change to the content of the WIC food packages since the programme's inception 40 years ago was implemented in October $2009^{(10)}$. Food packages were revised according to current US dietary guidelines to include low-fat milks, whole-grain breads and brown rice in addition to cash vouchers for purchasing fresh fruit and vegetables ${ }^{(10)}$. Compared with the previous WIC food packages, the new food packages are lower in saturated fat, cholesterol, total fat and sodium, and higher in fibre. In conjunction with the revisions in the food packages, stocking requirements for WIC-authorized vendors were also updated to meet these new requirements. Although the specifics of these renewed requirements vary by state, they generally included higher minimum stocking requirements for fresh fruit and vegetables, low- and reduced-fat milks and whole grains ${ }^{(10)}$. With more than 48000 WIC-authorized vendors across the country, this presents a substantial opportunity to reach a large number of people ${ }^{(11)}$.

WIC has achieved its success in improving dietary behaviour through nutrition education and by improving low-income households' access to nutritious foods. Historically this was viewed as economic access, the package being worth some \$US 44.98 per month ${ }^{(12)}$, but a sizeable amount of recent research has suggested that geographic access may be important for influencing consumer dietary behaviour $^{(13-22)}$. Residents in many low-income neighbourhoods need to travel long distances to access healthy foods, increasing their cost and reducing their likelihood of purchase. For example, residents of low-income neighbourhoods with limited access to grocery stores take on average $47 \%$ longer to access grocery stores than residents in higher-income areas with better access to stores $^{(23)}$. The challenge is even greater for the 2.3 million Americans who do not have access to a car and are more than 1 mile $(1.6 \mathrm{~km})$ away from their closest grocery store $^{(23)}$. For the new WIC food package to have a maximum impact, enough stores, especially small ones located in low-income neighbourhoods, would need to stay on the programme and agree to carry the new healthier options.

Overall, WIC vendors have been supportive of the food package changes ${ }^{(24)}$. Studies have begun to demonstrate changes in food store offerings before and after the revised policy was implemented. Increased availability of lowerfat milks, juice and whole grains was observed in WIC stores in two low-income neighbourhoods in Philadelphia, Pennsylvania between $2008 / 2009$ and $2010^{(25)}$. An assessment conducted within Hartford, Connecticut found that WIC-authorized vendors increased availability of fresh fruit and vegetables, lower-fat milks and whole grains between 2009 to $2010^{(26)}$, while another study in Connecticut found increased availability of whole-grain products throughout the state between spring 2009 and spring 2010 ${ }^{(27)}$. WIC vendors in northern Illinois increased fresh fruit and vegetable availability between 2008 and $2010^{(28)}$, while vegetable shelf space and availability of whole grains and reduced-fat milks increased in WIC stores in New Orleans, Louisiana between 2009 and $2010^{(29)}$. These studies underscore the complexity of the retail food environment even when the focus is just on WIC foods. In addition to availability of food, quality, price, shelf space and variety of food items were assessed in the studies above.

Although vendors have made many positive changes, results of a multi-site study revealed there were many initial barriers in adhering to the new stocking requirements including having a good relationship with a supplier for the required food items, ensuring the ongoing supply of fresh foods and explaining the new requirements to participants ${ }^{(24)}$. Another multi-site study identified a number of challenges in meeting the stocking requirements, including lack of appropriate infrastructure, supplier awareness and meeting specific package size and form of some required items ${ }^{(30)}$. The stocking requirements are indeed extensive. For example, in Louisiana, WIC vendors are required to carry eighteen different foods and a total of thirty-five different varieties (e.g. five varieties of whole-grain cereals).

As the WIC food package revisions become institutionalized, nutritionists, programme managers and policy makers need simple tools to monitor the progress of changes in the retail food environment. This is, in part, a compliance issue for WIC vendors, and better tools will enable an improved monitoring of this. But assessment of the retail environment also extends to non-WIC store operators who operate in the same communities. For example, the Philadelphia study cited above found changes in non-WIC stores, even though they were not required to make changes ${ }^{(25)}$. Understanding how stores operate in relation to WIC requirements provides a good benchmark for evaluating a community's retail food environment.

The current study describes the development of a new WIC Availability Index (WIC-AI) that was designed to be simple, easily understandable, transparent and applicable for both food environment researchers as well as public health practitioners to quickly assess and compare WICapproved food item availability. The index was tested with data collected from the retail food environment in New Orleans, Louisiana to examine differences between WIC and non-WIC stores and to examine compliance in WIC stores a year after package changes were instituted.

\section{Methods}

The WIC-AI was developed out of a larger natural experiment with pre-post test design examining the impact of WIC food package revisions on the food retail environment in New Orleans using the store as the unit of analysis. 
The store sample for the study was generated by merging a 2009 WIC vendor list obtained from the Louisiana state WIC department with our New Orleans food store census data in which we conduct annual observations of all food retail outlets in New Orleans ${ }^{(31)}$. All WIC and non-WIC stores were identified and categorized to include supermarkets (annual sales > \$US 5 million), medium (annual sales \$US 1-5 million) and small food stores (annual sales $<\$$ US 1 million). Of the 176 WIC and non-WIC supermarkets, medium and small food stores identified in our 2009 store census, 157 were visited by our survey teams and 139 (79 \%) were observed in September 2009, before the implementation of the WIC food package changes. Survey teams were not able to visit nineteen stores before time ran out on this data collection period. Any potential bias for not including information on these stores should be minimal because stores were approached in a random sequence. Eighteen stores did not grant permission for the store observation, mostly because the store manager was not present. Data were collected in the observed stores again in September and October of 2010, one year after the WIC food package change. A total of 128 stores were observed again in 2010 and used as the analytic sample, representing $92 \%$ of the 2009 sample.

An in-store survey previously developed and tested in New Orleans ${ }^{(32)}$ with inter-rater reliability measures $\geq 0.95^{(33,34)}$ was modified to include information on the availability of all categories of food items eligible for the Louisiana State WIC Program, except specialized milks such as lactose-free milk. The availability (yes/no) of fifty-six items was assessed based on programme requirements, which included specific brands for some foods (e.g. cereals, breads and rice), specific types for other foods (e.g. low-fat milks) and a range of varieties of other foods (e.g. fruits and vegetables). These items were contained in nine different sections within the survey instrument: Proteins, Whole
Grains, Cereals, Milk, Eggs and Cheese, Infant Formula and Cereal, Infant Fruit and Vegetables (jars of puréed fruit and vegetables), Juice and Fruit, and Vegetables. For example, within the Cereal section of the survey instrument, the presence (yes/no) of fifteen different varieties of cereals was assessed. Each of the fifteen varieties of cereals that were included in the survey instrument was specified as approved in the Louisiana State WIC Program vendor stocking requirements. For fruits and vegetables, we itemized those that were common to stores in New Orleans, and also recorded the availability of other fruits or vegetables that were not on our list. Graduate public health students and community project assistants were trained by Tulane staff on the proper protocol for in-store survey implementation. The permission of store operators or managers was obtained before data were collected onsite. The study did not involve human subjects and was exempt from Tulane institutional review board review.

\section{WIC Availability Index}

The WIC-AI was developed from the in-store survey as a summary score to assess the availability of all WIC foods. The WIC-AI was modelled on the Healthy Eating Index with total points ranging from 0 to 100 and encompassing ten different components ${ }^{(35,36)}$. The WIC-AI components and score are detailed in Table 1. Each of the ten components represents a category of WIC food package item (milk, grains, fruits, vegetables, etc.) and has a component score between 0 and 10. In defining the ten categories we attempted to develop a balanced index that would reflect weighting across dietary needs aligned with the US Department of Agriculture's Choose My Plate ${ }^{(37)}$ and Dietary Guidelines for Americans ${ }^{(38)}$ but also maintain enough disaggregation to represent the challenges at the retail level of carrying all these foods. For example, cereals and grains were grouped into one category as were milks

Table 1 WIC Availability Index (WIC-AI) scoring system

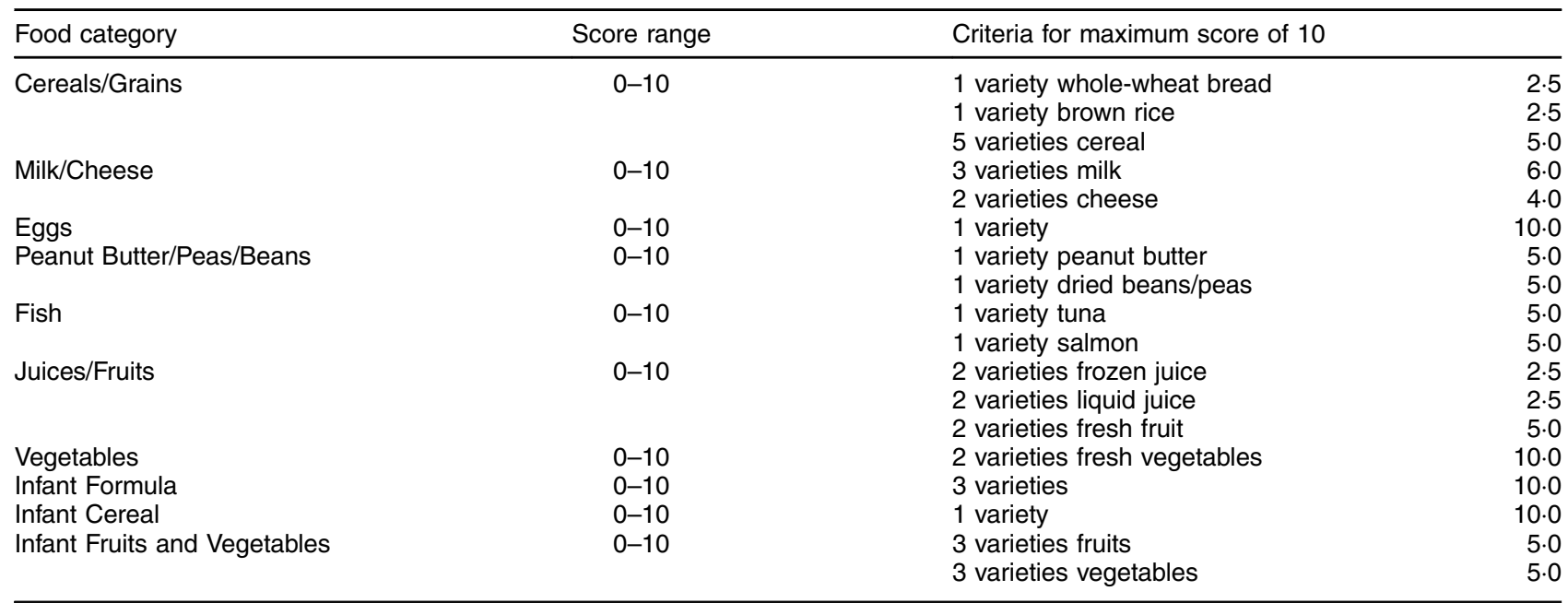

WIC, Special Supplemental Nutrition Program for Women, Infants, and Children. 
Table 2 Median WIC Availability Index (WIC-AI) by WIC vendor status and store type, New Orleans, LA, USA, 2009

\begin{tabular}{|c|c|c|c|c|c|c|c|c|c|}
\hline \multirow[b]{2}{*}{ Store type } & \multicolumn{3}{|c|}{ Overall } & \multicolumn{3}{|c|}{ Non-WIC } & \multicolumn{3}{|c|}{ WIC } \\
\hline & $n$ & Median & IQR & $n$ & Median & IQR & $n$ & Median & IQR \\
\hline Supermarket & 17 & $93 \cdot 3^{* *}$ & $11 \cdot 3$ & 6 & 93.5 & 7.9 & 11 & 93.3 & $12 \cdot 0$ \\
\hline Medium store & 18 & $69 \cdot 8^{\star \star}$ & $16 \cdot 8$ & 10 & $56 \cdot 8$ & 31.5 & 8 & 71.3 & 3.0 \\
\hline Small store & 93 & $48 \cdot 0^{\star *}$ & 35.5 & 66 & 38.0 & 28.0 & 27 & $66 \cdot 9^{\star *}$ & 8.1 \\
\hline
\end{tabular}

WIC, Special Supplemental Nutrition Program for Women, Infants, and Children; IQR, interquartile range.

${ }^{\star *} P<0.01$.

Table 3 Change in median WIC Availability Index (WIC-AI) of WIC authorized vendors by store type, New Orleans, LA, USA, 2009-2010

\begin{tabular}{lcccc}
\hline & \multicolumn{2}{c}{2009} & & \multicolumn{2}{c}{2010} \\
\cline { 2 - 3 } Store type & Median & IQR & Median & IQR \\
\hline Supermarket $(n 11)$ & 93.3 & $12 \cdot 0$ & $93 \cdot 3$ & $6 \cdot 6$ \\
Medium store $(n$ 8) & 71.3 & 3.0 & $86 \cdot 8^{\star \star}$ & $5 \cdot 3$ \\
Small store $(n$ 27) & 66.9 & $8 \cdot 1$ & $85 \cdot 5^{\star \star}$ & $8 \cdot 8$ \\
\hline
\end{tabular}

WIC, Special Supplemental Nutrition Program for Women, Infants, and Children; IQR, interquartile range. ${ }^{\star \star} P<0.01$.

and cheeses for nutrition reasons, but eggs, legumes and fish were categorized separately because of the different implications for supplying to and carrying in a store. This is particularly important to consider when applied to small food stores.

The ten components were then further divided and assigned subcomponent scores (e.g. infant fruits (0-5) and vegetables (0-5)). Subcomponent scores were based on the Louisiana State WIC Program requirements for varieties of specific food items. Stores were awarded points on each of these subcomponents based on availability of subcomponent varieties. A maximum score was given for meeting the state criteria, 0 points were given if the store did not carry a product, and a linear scale was used to assign points that partially met the criteria (e.g. three out of five varieties of cereals scored 3 points). Total points, after summing all subcomponent scores, ranged from 0 to 100 .

\section{Data analysis}

WIC-AI values were not normally distributed so median scores are reported rather than means. Wilcoxon signedrank tests were used to determine significant differences in WIC-AI values at baseline by store size and WIC vendor status as well as comparisons of baseline and follow-up values. Stores that were not measured in both 2009 and 2010 were excluded in the final analysis. All analyses were conducted using the statistical software package SAS version $9 \cdot 2$ (2008).

\section{Results}

Nearly all WIC stores participated at baseline (94\%) and the majority of non-WIC stores also participated ( $72 \%)$. As shown in Table 2, baseline results showed that the median
WIC-AI score was highest in supermarkets $(93 \cdot 3)$, followed subsequently by medium (69.8) and small food stores (48.0; $P<0 \cdot 01$ ). Examining each store type by WIC vendor status at baseline, there was no difference in median WICAI score for supermarkets. Although there was a larger difference in median WIC-AI score between the medium WIC (71.3) and non-WIC food stores (56.8), it was not statistically significantly at a value of $P<0 \cdot 05$. However, medium WIC food stores were significantly more likely to carry infant cereals than non-WIC medium food stores at baseline (data not shown). Small WIC stores (66.9) had significantly higher median overall WIC-AI score than small non-WIC stores $(38 \cdot 0)$ at baseline $(P<0 \cdot 01)$.

Follow-up in-store data from 2010 (Table 3) demonstrated very little change in median WIC-AI score for supermarkets regardless of WIC vendor status from 2009 to 2010. Significant increase in median WIC-AI score was observed from $2009(71 \cdot 3)$ to $2010(86 \cdot 8)$ in medium WIC stores $(P<0 \cdot 01)$. There was also a significant increase in median WIC-AI score from 2009 (66.9) to $2010(85.5)$ in small WIC food stores $(P<0.00001)$. Additionally, significant positive improvements were seen in small non-WIC $(P<0.05)$ stores from 2009 to 2010 (results not shown).

The components that showed change and helped contribute to this increase of overall WIC-AI score were examined in depth in small food stores, since these stores are least likely to carry healthy foods and are most commonly located in low-income neighbourhoods (Table 4). Cereals and grains increased in WIC stores from 2009 to $2010(P<0 \cdot 01)$. Juices and fruits increased in both WIC and non-WIC stores from 2009 to 2010 (both $P<0 \cdot 01$ ). Although non-WIC small food stores doubled the vegetable component score, this increase was not significant. The fourth WIC-AI component that demonstrated change was infant fruits and vegetables, which increased in WIC stores from 2009 to $2010(P<0 \cdot 01)$. 
Table 4 Change in median WIC Availability Index (WIC-AI) of small food stores by WIC vendor status, New Orleans, LA, USA, 2009-2010

\begin{tabular}{|c|c|c|c|c|c|c|c|c|}
\hline \multirow[b]{3}{*}{ Food category } & \multicolumn{4}{|c|}{ Non-WIC $(n$ 66) } & \multicolumn{4}{|c|}{ WIC ( $n$ 27) } \\
\hline & \multicolumn{2}{|c|}{2009} & \multicolumn{2}{|c|}{2010} & \multicolumn{2}{|c|}{2009} & \multicolumn{2}{|c|}{2010} \\
\hline & Median & IQR & Median & IQR & Median & IQR & Median & IQR \\
\hline Overall & 38.0 & 28.0 & $41 \cdot 6^{*}$ & $29 \cdot 0$ & 66.9 & $8 \cdot 1$ & $85 \cdot 5^{\star *}$ & 8.8 \\
\hline Cereals/Grains & $2 \cdot 8$ & 4.5 & 2.5 & 4.5 & 5.5 & 2.5 & $10 \cdot 0^{\star *}$ & 1.0 \\
\hline Milk/Cheese & $2 \cdot 0$ & $2 \cdot 0$ & $2 \cdot 0$ & 0.0 & $5 \cdot 0$ & $5 \cdot 0$ & $5 \cdot 0$ & $5 \cdot 0$ \\
\hline Eggs & $10 \cdot 0$ & $10 \cdot 0$ & $10 \cdot 0$ & $10 \cdot 0$ & $10 \cdot 0$ & 0.0 & $10 \cdot 0$ & 0.0 \\
\hline Peanut Butter/Peas/Beans & $10 \cdot 0$ & $5 \cdot 0$ & $10 \cdot 0$ & 5.0 & $10 \cdot 0$ & 0.0 & $10 \cdot 0$ & 0.0 \\
\hline Fish & $5 \cdot 0$ & $5 \cdot 0$ & $5 \cdot 0$ & 5.0 & $10 \cdot 0$ & $5 \cdot 0$ & $10 \cdot 0$ & 0.0 \\
\hline Juices/Fruit & 2.5 & 3.8 & $5 \cdot 0^{* *}$ & 5.0 & 7.5 & $5 \cdot 0$ & $8 \cdot 8^{\star *}$ & 2.5 \\
\hline Vegetables & $5 \cdot 0$ & $10 \cdot 0$ & $10 \cdot 0$ & $10 \cdot 0$ & $10 \cdot 0$ & $5 \cdot 0$ & $10 \cdot 0$ & 0.0 \\
\hline Infant Formula & 0.0 & 0.0 & 0.0 & 0.0 & 0.0 & $6 \cdot 6$ & 0.0 & $6 \cdot 6$ \\
\hline Infant Cereal & 0.0 & $10 \cdot 0$ & 0.0 & $10 \cdot 0$ & $10 \cdot 0$ & 0.0 & $10 \cdot 0$ & 0.0 \\
\hline Infant Fruit and Vegetables & 0.0 & 0.0 & 0.0 & 0.0 & 0.0 & 1.6 & $10 \cdot 0^{* *}$ & 1.7 \\
\hline
\end{tabular}

WIC, Special Supplemental Nutrition Program for Women, Infants, and Children; IQR, interquartile range.

${ }^{\star} P<0.05,{ }^{* *} P<0.01$.

\section{Discussion}

The present paper reports on the development and testing of a simple transparent monitoring tool for assessing the retail food store environment relative to the WIC programme's new food package requirements. Results of the study demonstrated that in New Orleans in 2009 median WIC-AI score was highest in supermarkets, followed by medium food stores and small food stores. No difference in median WIC-AI score was observed between supermarkets by WIC vendor status in 2009, although the median score was higher in small WIC stores than small non-WIC stores in 2009. No change in median WIC-AI score was observed among supermarkets from 2009 to 2010, largely due to ceiling effects as scores were already high in 2009. However, the median score increased from 2009 to 2010 in both medium and small WIC stores. The increase in WIC-AI score in the small WIC stores was attributed to increased availability of cereals/grains, juices/fruits and infant fruits and vegetables. Interestingly, small non-WIC stores also showed improvements in the juice/fruit category, and marginally in the vegetables category.

Supermarkets have the largest capacity to carry a variety of food items, followed by medium and small food stores so it is appropriate that before the updated WIC vendor guidelines were enforced, supermarkets scored highest followed by medium and small food stores on the WIC-AI. This may also explain why no difference was observed between WIC and non-WIC supermarkets and no change was observed from 2009 to 2010 among supermarkets. Before the revised guidelines were implemented, small WIC stores scored higher on the WIC-AI than small non-WIC stores. Increased scores were observed in both medium and small WIC stores from 2009 to 2010. This is consistent with other findings that WIC vendors carry more fresh fruit, lower-fat milks, and have greater availability of whole-grain bread and brown rice than non-WIC vendors, and healthier options overall, both before and after revisions to the WIC food package were implemented ${ }^{(25,26)}$. However, our data also demonstrated an increase in fruit and vegetable availability within small non-WIC stores. This may indicate that there is an increased demand among all consumers that the store owners are responding to.

Although it is difficult to compare our results directly with other studies, there is basic concordance with what is being found in the literature on the WIC food package change. Andreyeva and colleagues developed a Healthy Food Supply Score to summarize the availability, variety, quality and prices of healthy foods contained in the revised WIC food package ${ }^{(27)}$. Similar to results reported in the present paper, Andreyeva et al. found increased scores from 2009 to 2010, largely among WIC-authorized convenience and grocery stores, although a smaller increase was observed among non-WIC convenience and grocery stores. However, the increased scores observed in Connecticut were due largely to increased availability and variety of whole-grain products, while our analysis determined that increased availability of juices/fruits and infant fruits/ vegetables in addition to cereals/grains contributed to the increased scores within small WIC stores and juices/fruits and vegetables contributed within small non-WIC stores. Other investigators have also found store owners reported increased sales of and WIC recipients reported increase home availability and consumption of some of these same items shown to have increased in the retail food environment in New Orleans after the implementation of the revised WIC food packages ${ }^{(29,39-43)}$.

As with any index, detailed information is lost by reducing complex information into one number. Being one of the primary objectives of the study, the use of the tencomponent (and subcomponent) WIC-AI captured and summarized a multitude of retail food store environment information for easier comparison. However, in doing so, we not only categorized specific food items into groups based on a reflection of dietary needs but also to reflect the challenges for retailers to carry the food items. This was 
particularly important when examining the small food stores who were expected to have the largest challenges in accommodating the revised WIC stocking requirements.

A second limitation concerns the lack of information in the index on unhealthy food availability. Previous work has shown the importance of foods such as sodas, chips and candies to the overall food environment and their negative association with health outcomes such as weight status $^{(31)}$. However, our focus in the current paper was to develop a tool that could quantify the extent to which stores were adopting the new WIC guidelines, which do not include restrictions on non-WIC supported foods. Certainly an interesting topic for future research would be the impact of the WIC changes on the availability of unhealthy foods.

A relatively large sample of stores (79 \%) in New Orleans was studied here, so these findings are likely to be representative of the city's retail environment. Of the stores not observed, about half were due to survey time limitations and half were due to store refusals, but there is no reason to believe these stores are systematically different from stores in our sample.

As with all localized studies, results may not be generalizable to other cities, states and areas of the country. The WIC-AI was developed based on the Louisiana WIC requirements that meet federal guidelines. The specific foods authorized by state agencies do vary to allow substitute foods to accommodate cultural food preferences of local participants and characteristics of local food vendors ${ }^{(10)}$. However, all state requirements must meet the federal guidelines, and the major food categories are consistent across state agencies.

Andreyeva and colleagues developed a Healthy Food Supply Score to summarize information collected within food stores on availability, quality and price ${ }^{(27)}$. The WICAI is solely focused on availability, so the meaning of the summary score is more transparent. At an aggregate level, it provides an overall measure of the availability of WIC foods. Component scores can also be used to provide a measure of the availability of specific food groups. The WIC-AI scores would be easy to adapt for use in other cities, states or at a national level. The WIC-AI is a useful food environment research tool but could also be useful for other practitioners interested in examining their food retail environment contents. Researchers in California demonstrated the feasibility and usefulness of a general retail food store scoring system for public health practitioners in their $\mathrm{CX}^{3}$ Retail Food Store Score ${ }^{(44)}$. The WIC-AI could be adapted for use in other states by aligning the specific WIC-approved items to those allowed by the state agency. For example, in Louisiana canned tuna and salmon are accepted fish options whereas in other states, mackerel or sardines may also be approved. The survey tool and WIC-AI could be adapted to assess availability of the authorized food items particular to the state requirements.

\section{Conclusion}

In conclusion, the WIC-AI proved to be a simplified way to summarize complex food store environment data and was able to detect expected differences between WIC and non-WIC stores as well as differences between store sizes. Future research could include an examination of differential impact by various neighbourhood characteristics, particularly focused on those neighbourhoods considered to be 'food deserts' lacking access to full-service grocery stores. Research linking sales data with food consumption data should also be a high priority for the future. Additionally, monitoring sustained changes in food availability over time will inform investigations of the long-term impact of the WIC food package change on the neighbourhood food retail environment. Having a measure of progress across all stores allows for continued monitoring of improvements in access to healthy foods and a consideration of the potential for policy initiatives to improve access to healthier food options in a variety of store types and sizes.

\section{Acknowledgements}

Financial support: This work was supported by the Centers for Disease Control and Prevention (CDC), Atlanta, GA, through the Tulane Prevention Research Center (PRC) (Cooperative Agreement Number U48DP001948 including the Nutrition and Obesity Policy, Research and Evaluation Network); and the US Department of Agriculture (USDA)National Institute of Food and Agriculture (NIFA) (National Research Initiative \#2006-55215-16711). The funders had no role in the design, analysis or writing of this article. The findings and conclusions in this manuscript are those of the authors and do not necessarily represent the official position of the CDC or the USDA. Conflict of interest: None. Authorship: K.O. coordinated the 2010 data collection. B.G.L. conducted the statistical analysis of the data. L.F.D. coordinated 2009 and 2010 data collection and J.N.B. coordinated 2009 data collection. D.R. was the Principal Investigator for the study. All authors contributed to the writing of the manuscript. Ethics of buman subject participation: The study did not involve human subjects and was exempt from Tulane institutional review board review.

\section{References}

1. Oliveira V \& Frazao E (2009) The WIC Program: Background, Trends, and Economic Issues, 2009 Edition. Economic Research Report no. 73. Washington, DC: USDA, ERS.

2. Institute of Medicine, Committee to Review the WIC Food Packages (2006) WIC Food Packages: Time for a Change. Washington, DC: The National Academies Press.

3. Rose D, Habicht JP \& Devaney B (1998) Household participation in the Food Stamp and WIC programs increases the nutrient intakes of preschool children. $J$ Nutr $\mathbf{1 2 8}$ $548-555$ 
4. Fox MK, Hamilton WL \& Lin BH (editors) (2004) Effect of Food Assistance and Nutrition Programs on Nutrition and Health. vol. 3: Literature Review. Food Assistance and Nutrition Research Report no. 19-3. Washington, DC: USDA, ERS.

5. Colman S, Nochols-Barrer IP, Redline JE et al. (2012) Effects of the Special Supplemental Nutrition Program for Women, Infants, and Children (WIC): A Review of Recent Research. Report no. WIC-12-WM. Alexandria, VA: USDA, Food and Nutrition Service, Office of Research and Analysis.

6. Connor P, Bartlett S, Mendelson M et al. (2011) WIC Participant and Program Characteristics 2010. Report no. WIC-1-PC. Alexandria, VA: USDA, Food and Nutrition Service, Office of Research and Analysis.

7. Coleman-Jensen A, Nord M, Andrews M et al. (2011) Household Food Security in the United States in 2010. Economic Research Report no. ERR-125. Washington, DC: USDA, ERS.

8. Flegal KM, Carroll MD, Ogden CL et al. (2010) Prevalence and trends in obesity among US adults, 1999-2008. JAMA 303, 235-241.

9. Ogden CL, Carroll MD, Kit BK et al. (2012) Prevalence of obesity and trends in body mass index among US children and adolescents, 1999-2010. JAMA 307, 483-490.

10. Cole N, Jacobson J, Nichols-Barrer I et al. (2011) WIC Food Packages Policy Options Study. Alexandria, VA: USDA, Food and Nutrition Service, Office of Research and Analysis.

11. Gleason S, Pooler J, Bell L et al. (2013) 2013 WIC Vendor Management Study. Alexandria, VA: USDA, Food and Nutrition Service, Office of Policy Support.

12. US Department of Agriculture, Food and Nutrition Service (2013) WIC Program Participation and Costs 1974-2012. http://www.fns.usda.gov/pd/wisummary.htm (accessed August 2013).

13. Franco M, Diez-Roux AV, Nettleton JA et al. (2009) Availability of healthy foods and dietary patterns: the Multi-Ethnic Study of Atherosclerosis. Am J Clin Nutr 89, 897-904.

14. Bodor JN, Rose D, Farley TA et al. (2008) Neighbourhood fruit and vegetable availability and consumption: the role of small food stores in an urban environment. Public Health Nutr 11, 413-420.

15. Rose D \& Richards R (2004) Food store access and household fruit and vegetable use among participants in the US Food Stamp Program. Public Health Nutr 7, 1081-1088.

16. Morland K, Wing S \& Roux AD (2002) The contextual effect of the local food environment on residents' diets: the Atherosclerosis Risk in Communities Study. Am J Public Health 92, 1761-1767.

17. Blanchard T \& Lyson T (2003) Retail Concentration, Food Deserts, and Food Disadvantaged Communities in Rural America. Mississippi State, MS: Southern Rural Development Center.

18. Laraia BA, Siega-Riz AM, Kaufman JS et al. (2004) Proximity of supermarkets is positively associated with diet quality index for pregnancy. Prev Med 39, 869-875.

19. Moore LV, Diez Roux AV, Nettleton JA et al. (2008) Associations of the local food environment with diet quality - a comparison of assessments based on surveys and geographic information systems: the Multi-Ethnic Study of Atherosclerosis. Am J Epidemiol 167, 917-924.

20. Hickson DA, Diez Roux AV, Smith AE et al. (2011) Associations of fast food restaurant availability with dietary intake and weight among African Americans in the Jackson Heart Study, 2000-2004. Am J Public Health 101, Suppl. 1, S301-S309.

21. Moore LV, Diez Roux AV, Nettleton JA et al. (2009) Fast-food consumption, diet quality, and neighborhood exposure to fast food: the Multi-Ethnic Study of Atherosclerosis. Am J Epidemiol 170, 29-36.
22. Boone-Heinonen J, Gordon-Larsen P, Kiefe CI et al. (2011) Fast food restaurants and food stores: longitudinal associations with diet in young to middle-aged adults: the CARDIA study. Arch Intern Med 171, 1162-1170.

23. Ver Ploeg M, Breneman V, Farrigan T et al. (2009) Access to Affordable and Nutritious Food: Measuring and Understanding Food Deserts and Their Consequences: Report to Congress. Washington, DC: USDA, ERS.

24. Gittelsohn J, Laska MN, Andreyeva T et al. (2012) Small retailer perspectives of the 2009 Women, Infants and Children program food package changes. Am J Health Behav 36, 655-665.

25. Hillier A, McLaughlin J, Cannuscio CC et al. (2012) The impact of WIC food package changes on access to healthful food in 2 low-income urban neighborhoods. $J$ Nutr Educ Behav 44, 210-216.

26. Havens EK, Martin KS, Yan J et al. (2012) Federal nutrition program changes and healthy food availability. Am J Prev Med 43, 419-422.

27. Andreyeva T, Luedicke J, Middleton AE et al. (2012) Positive influence of the revised Special Supplemental Nutrition Program for Women, Infants, and Children food packages on access to healthy foods. J Acad Nutr Diet 112, 850-858.

28. Zenk SN, Odoms-Young A, Powell LM et al. (2012) Fruit and vegetable availability and selection: federal food package revisions, 2009. Am J Prev Med 43, 423-428.

29. Rose D, O'Malley K, Dunaway L et al. (2011) The influence of WIC changes on the retail food environment in New Orleans. Presented at the Annual Meeting of the American Society for Nutrition, Washington, DC, 10 April 2011.

30. Gleason S, Morgan R, Bell L et al. (2011) Impact of the Revised WIC Food Package on Small WIC Vendors: Insight from a Four-State Evaluation. Portland, ME: Altarum Institute.

31. Rose D, Hutchinson PL, Bodor JN et al. (2009) Neighborhood food environments and body mass index: the importance of in-store contents. Am J Prev Med 37, 214-219.

32. Farley T, Rice J, Bodor J et al. (2009) Measuring the food environment: shelf space of fruits, vegetables, and snack foods in stores. J Urban Health 86, 672-682.

33. Cohen DA, Schoeff D, Farley TA et al. (2007) Reliability of a store observation tool in measuring availability of alcohol and selected foods. J Urban Health 84, 807-813.

34. Miller C, Bodor JN \& Rose D (2012) Measuring the food environment: a systematic technique for characterizing food stores using display counts. J Environ Public Health 2012 , 707860 .

35. Kennedy ET, Ohls J, Carlson S et al. (1995) The Healthy Eating Index: design and applications. J Am Diet Assoc 95, 1103-1108.

36. Guenther PM, Reedy J \& Krebs-Smith SM (2008) Development of the Healthy Eating Index-2005. J Am Diet Assoc 108, 1896-1901.

37. US Department of Agriculture (2011) Food Groups Overview. http://www.choosemyplate.gov/food-groups/ (accessed January 2014).

38. US Department of Agriculture \& US Department of Health and Human Services (2010) Dietary Guidelines for Americans 2010. Washington, DC: US Government Printing Office.

39. Whaley SE, Ritchie LD, Spector P et al. (2012) Revised WIC food package improves diets of WIC families. J Nutr Educ Behav 44, 204-209.

40. Chiasson MA, Findley SE, Sekhobo JP et al. (2013) Changing WIC changes what children eat. Obesity (Silver Spring) 21, 1423-1429.

41. Odoms-Young AM, Kong A, Schiffer LA et al. (2014) Evaluating the initial impact of the revised Special Supplemental Nutrition Program for Women, Infants, and Children (WIC) food packages on dietary intake and home food 
availability in African-American and Hispanic families. Public Health Nutr 17, 83-93.

42. Ayala GX, Laska MN, Zenk SN et al. (2012) Stocking characteristics and perceived increases in sales among small food store managers/owners associated with the introduction of new food products approved by the Special Supplemental Nutrition Program for Women, Infants, and Children. Public Health Nutr 15, 1771-1779.
43. Andreyeva T, Middleton AE, Long MW et al. (2011) Food retailer practices, attitudes and beliefs about the supply of healthy foods. Public Health Nutr 14, 1024-1031.

44. Ghirardelli A, Quinn V \& Sugerman S (2011) Reliability of a retail food store survey and development of an accompanying retail scoring system to communicate survey findings and identify vendors for healthful food and marketing initiatives. $J$ Nutr Educ Behav 43, Suppl. 2, S104-S112. 